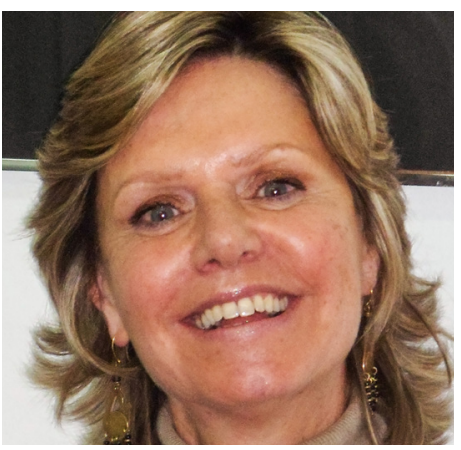

Denise Vaillant*

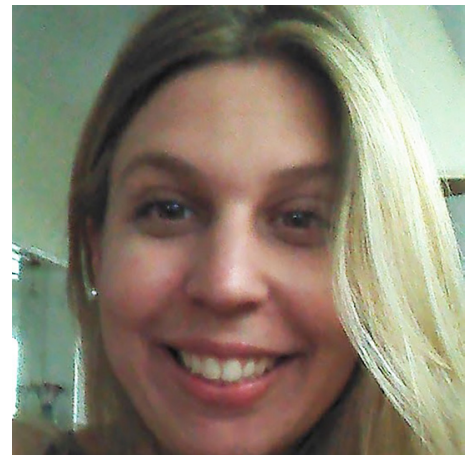

Lourdes Cardozo Gaibisso**

\title{
Desarrollo profesional docente: entre la proliferación conceptual y la escasa incidencia en la práctica de aula
}

\section{Teacher professional development: between the conceptual proliferation and the low incidence in classroom practice}

Recibido: 04-05-17

Aprobado: 21-05-17

\section{Resumen}

El Desarrollo Profesional Docente es una de las principales preocupaciones de la agenda educativa internacional. Existe consenso en que los buenos docentes y su desarrollo profesional constituyen un insumo clave para lograr la calidad educativa, también existe acuerdo en que, alterar las políticas del sector es un proceso complejo y de largo plazo que requiere voluntad gubernamental, capacidad técnica, continuidad de las políticas y amplio apoyo social (Vaillant, 2016). El presente artículo revisa la mejor evidencia disponible sobre las intervenciones en materia de desarrollo profesional docente en el periodo 2000-2016. En una etapa preliminar se consultó a informantes clave para afinar los ejes de la búsqueda bibliográfica. Luego se procedió a una exhaustiva revisión de bases de datos, artículos de investigación y libros. La búsqueda arrojó 384 documentos para codificar lo que nos permitió contar con un sustancioso cuerpo de conocimientos que podría ser útil a la hora de diseñar y llevar adelante intervenciones en materia de desarrollo profesional docente. Los resultados de la revisión de la literatura muestran que el DPD es entendido teóricamente de manera similar en los escritos en cuanto a su relevancia para mejorar las prácticas docentes. Sin embargo, los estudios evidencian también enorme diversidad en lo que respecta a la duración, el contenido y las modalidades de DPD. Sabemos mucho sobre qué hacer o por qué hacer, pero bastante menos sobre cómo hacer.

\footnotetext{
*Denise Vaillant: Posee un Doctorado en Educación de la Universidad de Québec à Montréal, Canadá y una Maestría en Planeamiento y Gestión Educativa de la Universidad de Ginebra, Suiza. Ocupó varios cargos de responsabilidad en la Administración de Educación Nacional de Educación Pública en Uruguay. Es profesora invitada en numerosas universidades latinoamericanas y europeas, asesora de varios organismos internacionales y autora de artículos y libros referidos a la temática de profesión docente, reforma e innovación educativas. Actualmente dirige el Instituto de Educación y el Programa de Doctorado en Educación de la Universidad ORT Uruguay. Es Investigadora Nivel II del Sistema Nacional de Investigadores (SNI) de Uruguay. Para contactar a la autora: vaillant@ort.edu.uy; www.denisevaillant.com
}

\footnotetext{
**Lourdes Cardozo-Gaibisso: Es Doctoranda en Educación, Lenguaje y Alfabetización (Universidad de Georgia, Estados Unidos), Master en Educación (Universidad ORT Uruguay) y Profesora de Educación Media en la especialidad inglés (Instituto de Profesores Artigas). Es Asistente de Investigación para la Fundación Nacional de Ciencias de Estados Unidos (Proyecto LISELL-B) y docente de postgrados del Instituto de Educación de la Universidad ORT Uruguay. Preside la Asociación Multilingüe y Multicultural del estado de Georgia, en Estados Unidos. Para contactar a la autora: lourdes@uga.edu
} 


\section{Abstract}

Teacher professional development is today one of the major concerns of the international education agenda. There is consensus that good teachers and their professional development are a key input to achieve quality education. There is also agreement that to alter educational policies is a complex and long-term process that requires governmental will, technical capacity, continuity, and broad social support (Vaillant, 2016). This article reviews the best available evidence on interventions in the field of teacher professional development during the period of 2000-2016. The first step to conduct this review was to consult key informants in order to refine the axes of the literature review. The literature review showed 384 documents, which enabled to build a substantial body of knowledge that could be useful to design and carry out interventions in the field of teacher professional development. Then an in-depth analysis of research articles and books followed.

\section{Palabras clave}

desarrollo profesional docente; prácticas de aula; políticas educativas; sostenibilidad; cambio en educación

\section{Keywords}

teacher professional development; classroom practices; educational policies; sustainability; educational change

\section{Introducción}

Un informe sobre la enseñanza en diversos países (Burns \& Darling-Hammond, 2014) identifica el desarrollo profesional docente como uno de los caminos para la mejora de la enseñanza y el aprendizaje tanto a nivel de la educación básica como de la educación superior. Durante décadas, los investigadores han insistido en que el conocimiento de los educadores es un componente clave para mejorar el éxito académico de los estudiantes (Lee, Luykx, Buxton \& Shaver, 2007). A pesar de este consenso, aún hay mucho por hacer para que el desarrollo profesional docente (DPD) esté integrado en las prácticas institucionales e individuales (McKenzie \& Santiago, 2005). Por otra parte, las actividades de DPD se identifican con frecuencia con talleres aislados y esporádicos que resultan inadecuados para producir cambios sostenibles en las prácticas de enseñanza (Boyle, White \& Boyle, 2004).

En sintonía con las críticas a las intervenciones de DPD, hace ya más de dos décadas, Darling-Hammond (1997), Lieberman (1996) y Darling-Hammond \& McLaughlin, (1995) reconocieron limitaciones en los programas de DPD, las que conservan hoy su vigencia. Tales limitaciones incluyen: la ausencia de participación activa por parte de los profesores, la falta de contextualización, y la corta duración de las intervenciones.

Es importante aclarar que, a pesar de estas limitaciones, organismos gubernamentales alrededor del mundo han invertido significativamente en programas de aprendizaje profesional para docentes. Al mismo tiempo, cabe constatar la ausencia de evaluación de esos programas y sus efectos reales sobre la enseñanza y el aprendizaje (Day \& Sachs, 2004; Garet, Porter, Desimone, Birman \& Yoon, 2001).
El mencionado consenso sobre la importancia del DPD, pero sus persistentes dificultades para generar y mantener modelos e intervenciones, hacen que este tema sea particularmente relevante en la agenda educativa contemporánea. Una de las razones para ello es que existe una fractura entre el copioso cuerpo de investigación sobre DPD y su influencia práctica y real en el aprendizaje de los docentes. De hecho, el DPD es uno de los temas educativos más complejos y relevantes, no porque no existan una multitud de estudios empíricos ni fundamentaciones teóricoconceptuales, sino porque a pesar de ser ampliamente investigado, ha fracasado consistentemente en producir resultados satisfactorios y sostenibles en el tiempo. Así, este artículo busca identificar y describir las tendencias en la investigación sobre DPD que surgen de la literatura a nivel internacional. Se indaga en la producción académica internacional en el ámbito del DPD durante el periodo 2000-2016, con la idea de que este conocimiento podría robustecer la investigación y las políticas docentes y mejorar los procesos de formación en servicio. De esta forma, se pretende desarrollar una comprensión más profunda de las intervenciones de DPD tratando de explorar las siguientes preguntas iniciales:

a) ¿Cuáles son los trabajos conceptuales y empíricos que marcan la literatura acerca del DPD en el periodo comprendido entre el 2000 y el 2016?

b) ¿Cuál es la mejor evidencia disponible acerca de intervenciones en desarrollo profesional docente respecto a: duración, frecuencia, componentes y enfoque?

c) ¿Qué componentes de DPD surgen de la literatura como indicadores $y$ factores

«Desarrollo profesional docente: entre la proliferación conceptual y la escasa incidencia en la práctica de aula», Denise Vaillant y Lourdes CardozoGaibisso. Cuaderno de Pedagogía Universitaria Vol. 13 / no. 26 / julio-diciembre 2016 / República Dominicana / PUCMM / ISSN $1814-4152$ (en línea) / 
predictivos del éxito y cambio de las prácticas educativas de profesores?

Las preguntas sirvieron de guía para identificar y analizar la documentación seleccionada y también para descubrir cuáles son las principales tendencias de la literatura examinada. En tal sentido, este estudio pretende abrir nuevos espacios de reflexión e investigación a través de la identificación de áreas subexploradas dentro del corpus existente.

La revisión de la literatura ha dejado en evidencia la escasa sistematización de los procesos de implementación e investigación en torno al DPD. Este artículo finaliza con una serie de recomendaciones y sugerencias prácticas que pretenden contribuir a la reflexión en torno al aprendizaje en servicio de los profesores, que podrá ser de utilidad, además, para la planificación e implementación de políticas educativas.

\section{Metodología}

Con el objetivo de delimitar el corpus de estudios que conforman esta revisión de la literatura se han seleccionado trabajos de investigación cualitativos, cuantitativos y de métodos mixtos; enmarcados en diversos paradigmas teóricos.

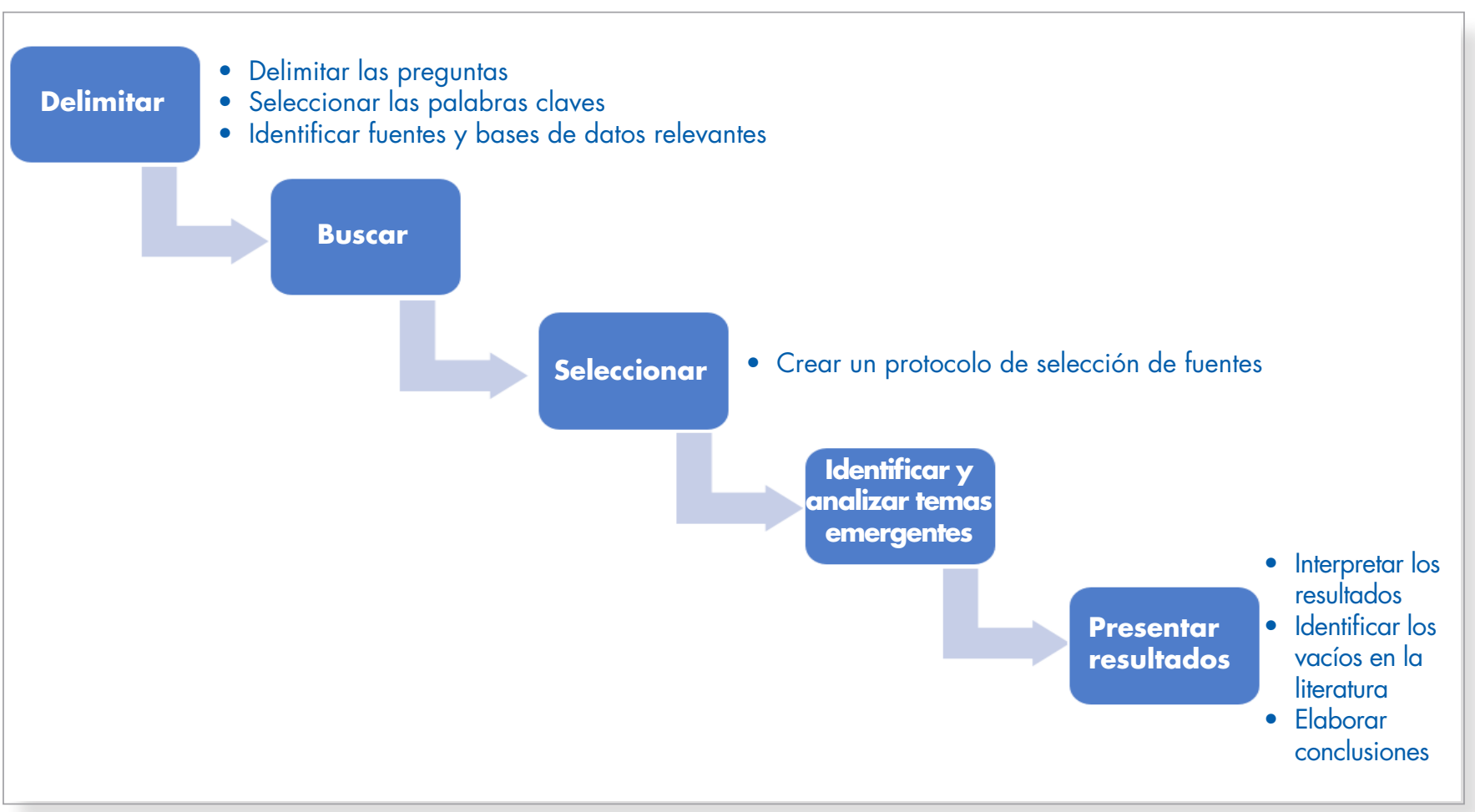

Figura 1 Procedimiento de definición del corpus

Para desarrollar un marco metodológico y diseñar un protocolo de selección y revisión de la literatura, hemos adaptado el diseñado por Wolfswinkel, Furtmueller \& Wilderom (2011) como se puede ver en la Figura 1. Una vez que se completaron las etapas iniciales de delimitación y búsqueda, y con el objetivo de analizar los estudios, se combinó el análisis temático (Vaismoradi, Jones, Turunen \& Snelgrove, 2016) con la práctica basada en la evidencia (PBE). Se seleccionó la PBE como metodología general, ya que el objetivo de esta revisión de la literatura no es sólo recopilar conocimientos teóricos acerca del DPD, sino también, y en última instancia, desarrollar conocimientos conceptuales y prácticos que guíen implementaciones futuras. La PBE es particularmente pertinente para esta revisión de la literatura porque es una metodología emergente que tiene como objetivo reunir evidencia empírica y científica que guíe futuras decisiones educativas (Cook \& Cook, 2016). Basadas en la noción de PBE, el protocolo de selección diseñado fue informado no sólo por las preguntas de investigación iniciales, sino también por la necesidad imperante de recopilar estudios que evidencien claras implicaciones para la práctica futura y el desarrollo de políticas educativas más eficaces y sostenibles.

Para llevar a cabo esta revisión de la literatura, se integraron dos fuentes principales. En primer lugar, las 
fuentes bibliográficas, artículos de investigación y libros seleccionados por las autoras a partir de entrevistas previas con informantes clave. En segundo lugar, se realizó una búsqueda en seis bases de datos que se describen a continuación: ERIC, Búsqueda Académica Completa, Investigación de Educación Completa, Abstracts de Administración de la Educación, Colección Sociológica y Colección de Desarrollo Profesional.

Como paso inicial, se realizó una búsqueda a través de las bases de datos nombradas previamente. Para los términos de búsqueda "Aprendizaje Profesional" o "Desarrollo Profesional", "Educación Media", "Educación Superior" entre el año 2000 y 2016 se obtuvo un total de 384 artículos de revistas académicas internacionales. Como se puede observar, se incorporaron bases de datos más allá del campo de la educación, ya que se considera potencialmente enriquecedor ampliar la mirada del DPD a otras disciplinas complementarias, en esta ocasión, Sociología y Administración. El número total de artículos incluidos se distribuyó de la siguiente manera:

Tabla 1

Distribución de artículos según base de datos y procedimiento de selección

\begin{tabular}{|l|c|c|c|c|}
\hline Base de datos & $\begin{array}{c}\text { Número de } \\
\text { articulos } \\
\text { inicial }\end{array}$ & $\begin{array}{c}\text { Primera } \\
\text { selección }\end{array}$ & $\begin{array}{c}\text { Segunda } \\
\text { selección } \\
\text { (PILR) }\end{array}$ & $\begin{array}{c}\text { Selección } \\
\text { final }\end{array}$ \\
\hline $\begin{array}{l}\text { Investigación de Educación } \\
\text { Completa }\end{array}$ & 158 & 35 & 22 & 15 \\
\hline Búsqueda Académica Completa & 90 & 11 & 5 & 1 \\
\hline Colección de Desarrollo Profesional & 69 & 2 & 1 & 1 \\
\hline ERIC & 34 & 5 & 1 & 3 \\
\hline $\begin{array}{l}\text { Abstracts de Administración } \\
\text { de la Educación }\end{array}$ & 26 & 0 & 0 & 0 \\
\hline Colección Sociológica & 7 & 0 & 0 & 0 \\
\hline Total & $\mathbf{3 8 4}$ & $\mathbf{5 3}$ & $\mathbf{2 9}$ & $\mathbf{2 0}$ \\
\hline
\end{tabular}

Con el fin de realizar una primera exploración general y obtener una cantidad razonable pero sustantiva de artículos que reflejaran los estudios realizados en el periodo 2000-2016, se realizó una evaluación inicial que consistió en analizar los resúmenes de cada artículo.

Mediante este procedimiento se descubrió que nueve artículos no coincidían con los términos de búsqueda iniciales, ya que se dirigían a una población objetivo diferente (educación primaria o de adultos); o habían utilizado el término desarrollo profesional de una manera genérica y abstracta, sin abordar explícitamente una intervención de aprendizaje profesional con claros resultados.

Con el objetivo de realizar un análisis eficaz y significativo de la literatura y obtener trabajos de investigación que se adhieran a los objetivos que nos hemos propuesto en primer plano, adaptamos el modelo denominado PILR (Población, Intervención, Lugar, Resultado) que consiste en una adaptación del modelo PISO, desarrollado por Booth \& Fry-Smith (2004). Al igual que con el modelo PISO, el marco de clasificación PILR permite a los investigadores ocuparse de cuestiones metodológicas y síntesis de hallazgos. Este marco asiste en la identificación manual de cuatro dimensiones clave en cada artículo, que permiten delimitar claramente los criterios de inclusión y exclusión.

Siguiendo este modelo, se elaboró un protocolo de clasificación que contribuyó a delimitar de forma transparente y rigurosa cuáles investigaciones se adaptaban a las cuatro dimensiones del marco de referencia. 
VENTANAS ABIERTAS A LA PEDAGOGÍA UNIVERSITARIA • 9

Tabla 2

PILR Criterios de Inclusión

\section{Dimensión}

Población

Intervención

Lugar

Resultado

\section{Criferio de inclusión}

Profesores de escuelas medias y educación superior en servicio de países tales como Estados Unidos, Grecia, Chile, Nueva Zelanda y Canadá.

Descripción de una intervención de DPD innovadora o adaptada.

Dentro y fuera del ámbito de educación formal.

Con referencia explícita al resultado de la intervención de DPD.
Se excluyeron de la muestra los artículos que no consistían en estudios sobre el DPD, presentados como artículos conceptuales o teóricos, así como aquellos que consistían en comparaciones de diferentes modelos, ya que no coincidían con el objetivo de esta revisión. En resumen, se seleccionaron aquellos estudios que tenían un enfoque explícito en el desarrollo y la implementación o en la investigación, evaluación o percepción de un programa de DPD.

\section{Resultados}

\section{Hacia una Definición de DPD: Conceptualización e Implementación}

En educación frecuentemente asistimos a un quiebre entre los saberes teóricos y las prácticas. El caso del DPD no es una excepción ya que, si bien es sabida la importancia del aprendizaje continuo de los docentes, muchas veces los saberes teóricos no se ven reflejados en las intervenciones planificadas y llevadas adelante. Un estudio realizado por Zecihner \& Tabachnick (1981) ya daba cuenta de que las nociones sobre enseñanza y aprendizaje de los docentes se desarrollan durante toda su trayectoria educativa y no solo durante su formación docente. Si bien, dicen los autores, durante la formación inicial los docentes tienden a ser innovadores en sus prácticas, durante sus primeros años de ejercicio, tienden a reproducir las prácticas educativas más conservadoras que vivieron durante su etapa escolar. Por este motivo, el DPD se convierte en una necesidad pedagógica para que los profesores puedan "mantenerse al ritmo del cambio y de revisar y renovar sus conocimientos, destrezas e ideas" (Day, 2005, p. 14).

Entre otros, la revisión de la literatura tuvo por objetivo identificar los estudios que conceptualizan el DPD. Es esencial reconocer en este punto que las investigaciones analizadas no presentan una definición única de DPD, aunque ofrecen sugerencias conceptuales sobre los supuestos que subyacen a sus componentes principales.

La literatura presenta gran diversidad, aunque muestra algunas características y entendimientos compartidos. En general, DPD constituye una intervención que se hace sobre los profesores y no con ellos; parecería que el valioso aporte de los docentes es frecuentemente ignorado. De igual manera, la evidencia (Garet, et al., 2001) sugiere que las intervenciones de corta duración suelen ser infructuosas. Entre los textos examinados, el estudio de DeFrance \& Fahrenbruck (2015) y el trabajo de Sahin \& Yildirim, (2016), reportan que la realización de breves intervenciones como talleres expositivos, no brindan a los docentes la oportunidad de involucrarse con el contenido a aprender.

La literatura muestra de forma consistente un modelo de DPD que es llevado adelante por coordinadores (Feighan \& Heeren, 2009; Munoz \& Guskey, 2009 Gross, 2010); y especialistas e investigadores universitarios (Fisher, Lapp, Flood \& Moore, 2006; August, Branum-Martin, Cárdenas-Hagan, Francis, Powell, Moore \& Haynes, 2007; Bansilal, Goba, Webb, James \& Khuzwayo, 2012; Richardson \& Janusheva, 2012; Buxton, Allexsaht-Snider, Kayumova, Aghasaleh, Choi \& Cohen, 2015; DeFrance \& Fahrenbruck, 2015; Pavez, Vergara, Santibanez \& Cofre, 2016). De acuerdo a esos textos, parecería que existe un modelo jerárquico en materia de DPD en el cual, son los especialistas los que implementan la formación de los docentes.

El DPD es entendido teóricamente de manera similar por los investigadores en cuanto a su relevancia para mejorar las prácticas docentes, y en consecuencia los aprendizajes de los estudiantes. No obstante, existen diferencias radicales cuando 
se trata de su implementación. Las investigaciones mostraron disparidades en términos de duración, contenido y conceptualización del aprendizaje del profesorado.

Algunos estudios identifican claramente qué componentes y prácticas hacen que una intervención de DPD sea exitosa (Desimone, 2009; Garet et al., 2001). Otros autores (Bolam \& McMahon, 2004) Ilaman la atención acerca de la falta de un cuerpo coherente de investigación que permita llegar a conclusiones o generalizaciones sobre el DPD.

Esa situación, podría explicarse por condiciones externas. Factores tales como las características específicas de cada contexto, la ubicación del centro educativo y la población objetivo, tanto de estudiantes como de profesores podrían explicar esta variabilidad.

\section{La Participación de los Docentes en el Desarrollo Profesional}

Los modelos tradicionales de DPD lo conceptualizan como una actividad que tiene como objetivo ampliar la formación inicial de profesores, y de igual modo proveerles de nuevos e innovadores conocimientos profesionales. (McKenzie \& Santiago, 2005). Esta perspectiva, bastante frecuente aún en la literatura, considera las actividades de DPD como una intervención externa a las instituciones educativas y que tiene como objetivo cambiar las prácticas de los docentes.

Producir y sostener cambios en estas prácticas educativas no sucede simplemente diciendo a los docentes qué y cómo enseñar. Los profesores deben tener un rol activo y participativo. Para que esto suceda, deben adquirir ricos conocimientos del área que enseñan, así como de pedagogía general y pedagogía específica. Y, además, deben desarrollar nuevas creencias respecto a su práctica, derribando muchas veces prácticas basadas en creencias intuitivas (Borko \& Puntman, 1995).

La literatura (Buxton et al., 2015; Thibodeau, 2008) enfatiza y confirma la participación de los docentes en su DPD como central. Buxton \& colegas (2015) sostienen que los docentes se relacionan con su desarrollo profesional de dos maneras. Por un lado, con su participación y asistencia a las actividades de formación; y por otro con la adaptación y aplicación de los contenidos y procedimientos aprendidos. En este sentido, los autores parten del entendimiento de que los docentes toman decisiones de manera permanente. Los autores reconocen que el contenido de la intervención no necesariamente se traducirá o aplicará de una sola manera, sino que los profesores tomarán decisiones sobre qué y cómo aplicar lo que aprendieron.
En síntesis, según la literatura, independientemente de las intervenciones de aprendizaje profesional a las que asisten, los profesores tienen una autonomía importante a la hora de aplicar lo aprendido. Sin embargo, son escasos los ejemplos de intervenciones en DPD los cuales se registra autonomía y oportunidades de toma de decisiones por parte de los docentes.

\section{Teorías explícitas de Aprendizaje en el DPD}

El aprendizaje puede ser definido como un proceso en el cual se internalizan elementos externos, que luego son aplicados por quien aprende (Scott \& Evans 2015). Lo que varía es el proceso entre esa mediación interna y externa del conocimiento y cómo diversas teorías (por ejemplo, el conductismo, el constructivismo y la teoría sociocultural) lo comprenden y lo conceptualizan. Uno de los objetivos de esta revisión de la literatura fue buscar referencias explícitas a las teorías del aprendizaje en los estudios seleccionados.

Un hallazgo clave ha sido la ausencia de referencia a las teorías de aprendizaje en servicio de los docentes. En algunos casos, los artículos establecieron paralelismos entre los programas de formación inicial de docentes y su formación en servicio, mostrando estos últimos como una extensión de los primeros. Eso significa que la literatura sobre DPD aún tiene pendiente la delimitación y diferenciación de la formación inicial docente y la formación de docentes en servicio. Una tendencia consistente de la literatura es presentar modelos de intervenciones de DPD como cursos que pretenden actualizar y capacitar a los profesores (Bansilal, Goba, Webb, James \& Khuzwayo, 2012).

Fisher et al. (2006) llevaron adelante una intervención que mostró que el aprendizaje docente consiste en transferir el conocimiento de expertos a profesores, con el objetivo de equipar a estos últimos con un nuevo conjunto de habilidades de instrucción. Este artículo, así como otros que también adoptaron un enfoque tradicional de aprendizaje, parecieran asemejarse a los modelos de educación bancaria, tan cuestionados en América Latina (Freire, 2000). Esta conceptualización simplificada del aprendizaje del profesorado, como depósito del conocimiento, tiene poco que ver con la compleja forma en que los docentes adquieren y desarrollan el conocimiento. Darling-Hammond (1997), por ejemplo, desarrolló esta complejidad, afirmando que primero, los profesores tienen que entender el contenido que van a enseñar y que necesitan algo más que entender los conceptos básicos de la instrucción o los procedimientos de enseñanza. Los profesores, afirma, necesitan aplicar sus conocimientos de manera flexible para resolver los problemas inesperados que puedan surgir en el contexto de aprendizaje. En sintonía con estas afirmaciones, Shulman (1987) también se 
adhiere a esta complejidad y afirma que la comunidad pedagógica en general necesita desarrollar un entendimiento más amplio acerca de los saberes que se necesitan para enseñar, de dónde provienen esos saberes y cómo se vinculan con los procesos de enseñanza y aprendizaje.

La literatura refiere muy poco a las características del aprendizaje de adultos. También son pocas las referencias al vínculo entre el profesor y el cambio institucional. El conocimiento se conceptualiza como transferible y el aprendizaje de adultos, con sus particularidades, no es abordado explícitamente en los escritos examinados.

Las intervenciones de DPD examinadas en la revisión de la literatura se implementan en diversos contextos y entornos. Siguiendo la clasificación de DPD que hace Lieberman (1996), se identifican en los escritos examinados:

- intervenciones denominadas por Lieberman (1996) de "aprendizaje directo" realizadas en instituciones educativas, a través de conferencias, talleres, seminarios y charlas, generalmente de corta duración;

- intervenciones en la institución educativa identificadas en 10 de los artículos revisados efectuadas dentro del ámbito educativo formal con el apoyo de capacitadores, mentores y pares;

- intervenciones fuera del centro educativo implementadas a través de alianzas con universidades locales o especialistas;

- intervenciones que combinan los escenarios previamente descritos e identificados como beneficiosos para el aprendizaje docente (Day \& Sachs, 2004).

La mayoría de los artículos examinados confirman que ninguna institución educativa es una isla, que los docentes no aprenden ni se desarrollan profesionalmente aislados, y que los profesores aprenden en una multitud de escenarios, realizando diferentes actividades y tareas. Sin embargo, sólo tres estudios proponen una combinación de diversas actividades de aprendizaje profesional desarrolladas en un periodo de un año.

Un estudio de Buxton y colaboradores (2015) presentó un modelo de DPD que involucró cuatro contextos para el aprendizaje profesional: un instituto de aprendizaje profesional de verano, observaciones en el aula, talleres de profesores, estudiantes y sus familias, y talleres de aprendizaje para docentes. Del mismo modo, August y sus colegas (2014) implementaron un modelo de DPD que combinaba talleres tradicionales de profesores, pero también agregaba un componente de tutoría y observación quincenal.

Parecería que en los últimos años se ha ampliado de forma significativa la comprensión de qué es DPD y dónde puede tener lugar, expandiendo los ambientes más tradicionales fuera de las instituciones educativas.

\section{La sostenibilidad de la Intervención: ¿Qué sucede cuando el DPD culmina?}

Otra de las preguntas que guiaron la revisión de la literatura, refiere a qué elementos o condiciones contribuyen a la sostenibilidad de una intervención en el tiempo. En los escritos examinados, cinco estudios hicieron referencia a cambios estructurales y contextuales que podrían influenciar potencialmente la sostenibilidad de una intervención. Por ejemplo, May (2007) afirmó que las demandas externas constituyen frenos para que las iniciativas de DPD prosperen en el tiempo. Una posible solución para esto sería para May (2007) que las instituciones educativas permanezcan involucradas en la intervención durante períodos de tiempo prolongados (mínimo un año) que permitan mayor exposición al aprendizaje profesional. Además, sugiere un exhaustivo seguimiento de los resultados de las intervenciones de DPD a mediano y largo plazo.

En esta línea, un estudio de Pradl (2002) mostró el impacto de una intervención de DPD en un centro educativo en la tasa de graduación universitaria 10 años después de iniciada la intervención.

Whitehead (2010) en un estudio, recoge información de una intervención un año después de su finalización. La evidencia de ese estudio sugiere que la combinación de un enfoque específico en el área académica que se pretende mejorar, junto con una sólida base teórica y trabajo práctico llevado a cabo con los profesores, pueden aumentar las probabilidades de sostenibilidad.

Parecería que la literatura tiende a centrar su atención en los resultados concretos y a corto plazo de la intervención de DPD, y no en su permanencia en el tiempo que es lo que produce efectos duraderos y profundos en las instituciones educativas.

\section{Discusión}

La revisión de la literatura evidencia, de acuerdo a Wanzek, Vaughn, Scammacca, Gatlin, Walker \& Capin (2016), que sabemos mucho sobre qué hacer o por qué hacer, pero bastante menos sobre cómo hacer. Hemos intentando obtener una mejor comprensión del DPD a través de la identificación de temas emergentes en la literatura internacional reciente. Las preguntas que hemos planteado, desde lo 
conceptual y teórico, buscaban dar respuesta a aquellos aspectos prácticos que llevan al éxito o fracaso de una intervención en materia de DPD.

No existe en la literatura una definición clara acerca del DPD y de cómo debe ser implementado y evaluado. Lo que ha puesto de manifiesto la revisión realizada (Day \& Sachs, 2003), es que el DPD se implementa y ejecuta en una multitud de formas y entornos, e involucra a muchos actores educativos como directores, coordinadores y administradores, dentro y fuera de los ámbitos formales de aprendizaje de docentes en servicio. Estos ámbitos pueden incluir talleres con especialistas, intercambios entre pares, entrenamientos de verano, entre muchos otros.

La identificación dentro de la literatura, que muestra el DPD como una actividad explícita y definida por su intencionalidad, puede ser cuestionada a la luz de nuevas conceptualizaciones. Borko (2004), por ejemplo, señala que el aprendizaje profesional de los docentes ocurre de forma permanente, dentro de contextos formales, pero también en las interacciones cotidianas con colegas y estudiantes. Esta nueva visión de DPD puede desafiar profundamente no sólo su tejido conceptual, sino también qué actividades cuentan como DPD, y cómo las mismas son evaluadas. En este sentido, los límites del DPD no son tan claros, y la medición o evaluación del impacto de una intervención se vuelve entonces más compleja.

En lo que respecta a cuáles son los factores que contribuyen al éxito del DPD, la literatura mostró que la participación de los profesores y la oportunidad de que los mismos tomen decisiones en cuanto a su aprendizaje profesional, tiene un impacto positivo. Sin embargo, una pregunta que quedó sin respuesta, es qué elementos hacen sostenible una intervención.

En este sentido, los estudios revisados hacen poca o ninguna referencia a los cambios estructurales que se pueden mantener a lo largo del tiempo, lo que demuestra que los escritos examinados han medido escasamente el impacto a largo plazo.

En consonancia con esta falta de sostenibilidad en el tiempo, un elemento adicional que surge de la literatura es la tendencia a conceptualizar y ejecutar las intervenciones de DPD como eventos aislados y de corta duración.

En conclusión, la literatura ofrece un cuadro complejo y contradictorio para los investigadores. Por un lado, los estudios parecen haber llegado a un consenso sobre la importancia del DPD, pero, por otro lado, existen pocos datos acerca de los problemas de implementación, evaluación y sostenibilidad, y acerca de las voces docentes.
La literatura revisada nos brinda una serie de pista para pensar el DPD, entre las cuales se encuentran:

- Ampliar la noción de DPD a contextos más informales y cotidianos. En este sentido, los investigadores y los formadores de docentes en servicio, deben tener en cuenta las múltiples oportunidades de auto-aprendizaje que tienen los profesores.

- Abandonar la premisa de DPD como algo que puede ocurrir de manera rápida. Entender que el aprendizaje de docentes en servicio y el cambio en las prácticas requieren tiempo, tanto para ser efectivos como sostenibles.

- Re-conceptualizar las iniciativas de DPD como un esfuerzo cuyo éxito depende de la participación de varias partes interesadas, y reconocer la necesidad de crear conexiones duraderas no sólo con los docentes, sino también con los directores, coordinadores y otros actores institucionales.

- Centrarse en el desarrollo de intervenciones de DPD que comprendan las complejidades del aprendizaje de adultos.

- Crear intervenciones contextualizadas y específicas, de acuerdo a las características de cada institución o grupo de docentes.

- Fomentar el desarrollo de recursos flexibles y materiales didácticos como parte de cualquier intervención. Orientar a los profesores a comprender las conexiones prácticas del contenido que se les presenta.

- Desarrollar intervenciones de DPD que tengan lugar en escenarios diversos.

- Conceptualizar el DPD no sólo como una oportunidad para aprender sobre contenidos de enseñanza, sino también como una oportunidad para intercambiar y reflexionar acerca de las finalidades de la educación y del papel de las instituciones educativas en la sociedad.

La revisión de la literatura presentada en este artículo nos dio la oportunidad de identificar los temas emergentes y las brechas de investigación. Pero aún es mucho lo que queda por investigar acerca del "cómo hacer" para que el DPD tenga incidencia en las aulas y en la actuación del docente a largo plazo. 


\section{Referencias}

August, D., Branum-Martin, L., Cárdenas-Hagan, E., Francis, D. J., Powell, J., Moore, S., \& Haynes, E. F. (2014). Helping ELLs meet the Common Core State Standards for literacy in science: The impact of an instructional intervention focused on academic language. Journal of Research on Educational Effectiveness, 7(1), 54-82.

Bansilal, S., Goba, B., Webb, L., James, A., \& Khuzwayo, H. (2012). Tracing the impact: A case of a professional development programme in Mathematical Literacy. Africa Education Review, 9(sup1), S106-S120.

Booth, A., \& Fry-Smith, A. (2004). Developing the research question. E-text on health technology assessment (HTA) information resources. Retrieved from https://www.nlm.nih.gov/archive/20060905/ nichsr/ehta/chapter2.html

Borko, H. (2004). Professional development and teacher learning: Mapping the terrain. Educational researcher, 33(8), 3-15.

Borko, H., \& Putnam, R. T. (1995). Expanding a teacher's knowledge base: A cognitive psychological perspective on professional development. In Guskey \& Huberman (Eds.) Professional development in education: New paradigms and practices (35-65). New York: Teachers College Records

Boyle, B., While, D., \& Boyle, T. (2004). A longitudinal study of teacher change: What makes professional development effective? The Curriculum Journal, 15(1), 45-68.

Burns, D., \& Darling-Hammond, L. (2014). Teaching around the world: What can TALIS tell us. Retrieved from https://edpolicy.stanford.edu/sites/default/ files/publications/teaching-around-world-what-cantalis-tell-us_3.pdf

Buxton, C. A., Allexsaht-Snider, M., Kayumova, S., Aghasaleh, R., Choi, Y. J., \& Cohen, A. (2015). Teacher agency and professional learning: Rethinking fidelity of implementation as multiplicities of enactment. Journal of Research in Science Teaching, 52(4), 489502.
Cook, B. G., \& Cook, L. (2016). Leveraging EvidenceBased Practice through Partnerships Based on Practice-Based Evidence. Learning Disabilities--A Contemporary Journal, 14(2).

Darling-Hammond, L. (1997). The Right To Learn: A Blueprint for Creating Schools That Work. The Jossey-Bass Education Series.

Darling-Hammond, L. (2014). Can value added add value to teacher evaluation? Educational Researcher, 44(2), 132-137.

Day, C., \& Sachs, J. (2004). Professionalism, performativity and empowerment: discourses in the politics, policies and purposes of continuing professional development. In Day \& Sachs (Eds.) International handbook on the continuing professional development of teachers (3-32) Berkshire: Open University Press

Day, C. (2005). Formar docentes. Cómo, cuándo y en qué condiciones aprende el profesorado. Madrid, Narcea.

DeFrance, N. L., \& Fahrenbruck, M. L. (2015). Constructing a Plan for Text-Based Discussion. Journal of Adolescent \& Adult Literacy.

Desimone, L. M. (2009). Improving impact studies of teachers' professional development: Toward better conceptualizations and measures. Educational researcher, 38(3), 181-199.

Desimone, L. M., Porter, A. C., Garet, M. S., Yoon, K. S., \& Birman, B. F. (2001). Effects of professional development on teachers' instruction: Results from a three-year longitudinal study. Educational evaluation and policy analysis, 24(2), 81-112.

Feighan, K., \& Heeren, E. (2009). She was my backbone: Measuring coaching work and its impact. CEDER Yearbook, 67-93.

Fisher, D., Lapp, D., Flood, J., \& Moore, K. (2006). Linking literacy teaching with assessment: a continuing professional development initiative for secondary schools. Literacy, 40(2), 115-122.

Freire, P. (2000). Pedagogy of the oppressed. New York: Continuum, c2000. 
Garet, M. S., Porter, A. C., Desimone, L., Birman, B. F., \& Yoon, K. S. (2001). What makes professional development effective? Results from a national sample of teachers. American educational research journal, 38(4), 915-945

Gross, P. A. (2010). Not another trend: Secondary-level literacycoaching. The Clearing House, 83(4), 133-137.

Lee, O., Luykx, A., Buxton, C., \& Shaver, A. (2007). The challenge of altering elementary school teachers' beliefs and practices regarding linguistic and cultural diversity in science instruction. Journal of Research in Science Teaching, 44(9), 1269-1291.

Lieberman, A. (1996). Creating intentional learning communities. Educational Leadership, 54(3), 51-55.

May, S. (2007). Sustaining effective literacy practices over time in secondary schools: School organisational and change issues. Language and Education, 21(5), 387-405.

McKenzie, P., \& Santiago, P. (2005). Teachers matter: attracting, developing and retaining effective teachers. Paris: Organisation for Economic Cooperation and Development, c2005.

Muñoz, M. A., Guskey, T. R., \& Aberli, J. R. (2009). Struggling readers in urban high schools: Evaluating the impact of professional development in literacy. Planning and Changing, 40(1/2), 61.

Pavez, J. M., Vergara, C. A., Santibañez, D., \& Cofré, H. (2016). Using a Professional Development Program for Enhancing Chilean Biology Teachers' Understanding of Nature of Science (NOS) and Their Perceptions About Using History of Science to Teach NOS. Science \& Education, 25(3-4), 383-405.

Pradl, G. M. (2002). Linking Instructional Intervention and Professional Development: Using the Ideas behind Puente High School English to Inform Educational Policy. Educational Policy, 16(4), 522-546.

Richardson, J., \& Janusheva, V. (2012). The Lasting Impact of" Reading to Learn" and Sustainability of Professional Development. International Journal of Learning, 18(9).
Scott, D., \& Evans, C. (2015). The elements of a learning environment. In Scott \& Hargreaves (Eds.) In The SAGE Handbook of Learning (pp 189-202). Los Angeles: SAGE Publications Ltd.

Shulman, L. (1987). Knowledge and teaching: Foundations of the new reform. Harvard educational review, 57(1), 1-23.

Thibodeau, G. M. (2008). A content literacy collaborative study group: High school teachers take charge of their professional learning. Journal of Adolescent \& Adult Literacy, 52(1), 54-64.

Vaillant, D. (2016). El fortalecimiento del desarrollo profesional docente: una mirada desde Latinoamérica. Journal of Supranational Policies of Education (JoSPoE). 5 (2), 5-21.

Vaismoradi, M., Jones, J., Turunen, H., \& Snelgrove, S. (2016). Theme development in qualitative content analysis and thematic analysis. Journal of Nursing Education and Practice, 6(5), 100.

Wanzek, J., Vaughn, S., Scammacca, N., Gatlin, B., Walker, M. A., \& Capin, P. (2016). Meta-Analyses of the Effects of Tier 2 Type Reading Interventions in Grades K-3. Educational Psychology Review, 28(3), 551-576. http://doi.org/10.1007/s10648-0159321-7

Whitehead, D. (2010). The year after: sustaining the effects of literacy professional development in New Zealand secondary schools. Language and Education, 24(2), 133-149.

Wolfswinkel, J., Furtmueller, E., \& Wilderom, C. (n.d). Using grounded theory as a method for rigorously reviewing literature. European Journal Of Information Systems, 22(1), 45-55 